\title{
Aspectos microbiológicos de las nuevas quinolonas: levofloxacina, sparfloxacina, trovafloxacina y grepafloxacina
}

\author{
OLIVIA TRUCCO A. ${ }^{1}$
}

\author{
MICROBIOLOGICAL ASPECTS OF THE NEW QUINOLONES: \\ LEVOFLOXACIN, SPARFLOXACIN, TROVAFLOXACIN AND \\ GREPAFLOXACIN
}

The new "third generation quinolones": levofloxacin, sparfloxacin, trovafloxacin and grepafloxacin are more active in vitro than previous quinolone generations against Streptococcus pneumoniae, Streptococcus pyogenes and methicillin sensitive Staphylococcus aureus, they are active against Gram negative enteric bacilli (but less than ciprofloxacin), and against atypical pneumonia agents (Mycoplasma pneumoniae, Chlamydia pneumoniae, Legionella pneumoniae); besides, some of these molecules (trovafloxacin, sparfloxacin) have in vitro activity against anaerobes species. Considering their broad spectrum of therapy activity, the new quinolones represent a major progress in anti infective therapy for ambulatory and hospitalized patients.

Cientos de quinolonas han sido sintetizadas y evaluadas en las últimas tres décadas. El miembro original del grupo, introducido en el mercado farmacéutico hace ya 36 años, ácido nalidixico, de restringida actividad antibacteriana sobre enterobacterias, particularmente sobre Escherichia coli, permaneció como un antibiótico útil para combatir infecciones de la vía urinaria -de uso oral- por su espectro, pobre nivel sérico, y excelente excreción urinaria. Este, junto a ácido oxolínico y pipemídico, quienes demostraron además muy pobre actividad frente a Pseudomonas aeruginosa, constituyeron las quinolonas de primera gene- ración, reservadas para infecciones urinarias.

La incorporación en la década de los 80 de fluor en la molécula, creó las quinolonas de segunda generación. El primer compuesto de este grupo, norfloxacina, más activo microbiológicamente que sus predecesores, por su mala absorción oral y baja concentración plasmática, se mantiene también restringido a la vía urinaria.

La introducción de nuevos radicales en esta molécula creó las siguientes quinolonas: pefloxacina, ofloxacina, enoxacina y ciprofloxacina (la más activa del grupo), las que lograron, además de mejorar la absorción oral y la dis-

\footnotetext{
${ }^{1}$ Instituto de Ciencias Biomédicas, Facultad de Medicina, Universidad de Chile
} 
Aspectos microbiológicos de las nuevas quinolonas - O. Trucco A.

tribución sistémica, excelente actividad antimicrobiana in vitro sobre enterobacterias, con CIMs menores o iguales a $0,25 \mu \mathrm{g} / \mathrm{ml}$. En bacilos Gram negativos no fermentadores presentaban variada actividad antimicrobiana.

Sobre patógenos respiratorios Haemophilus influenzae, Moraxella catarrhalis, Legionella pneumophila y Mycoplasma pneumoniae, demostraron buena actividad in vitro. En bacterias Gram positivas como Staphylococcus aureus y estafilococos coagulasa negativa, éstas eran activas, no así en especies de Streptococcus donde o carecían de actividad antimicrobiana o ella era límite. Finalmente también carecían de actividad sobre bacterias anaerobias. Nuevas modificaciones dieron origen a lomefloxacina, fleroxacina, temafloxacina y tosufloxacina, mejorando en algunas de ellas la vida media. ${ }^{1}$

Grandes esfuerzos por sintetizar nuevos compuestos que optimizaran la actividad biológica de las quinolonas, particularmente sobre bacterias Gram positivas y anaerobias, prolongaran su vida media, disminuyeran los efectos adversos y su interacción con otros fármacos, generaron las llamadas quinolonas de tercera generación: levofloxacina, sparfloxacina, trovafloxacina, clinafloxacina, gatifloxacina, grepafloxacina y moxifloxacina.

\section{¿Por qué la necesidad de nuevas quinolonas?}

Desde un punto de vista microbiológico las fluoroquinolonas disponibles demostraban falencias que debían ser corregidas:

- agregar actividad frente a cocáceas Gram positivas como Streptococcus pneumoniae, Streptococcus grupo viridans, Enterococcus $s p$.

- mejorar la actividad frente a Staphylococcus, principalmente resistentes a meticilina.

- mejorar la actividad sobre bacterias "atípicas" (Mycoplasma sp, Chlamydia sp, Legionella sp).

- agregar actividad sobre bacterias anaerobias, particularmente Bacteroides spp., Prevotella spp. y otras

- mantener o mejorar la actividad antibacteriana frente a bacilos Gram negativos, (enterobacterias y no fermentadores), y aquellos de difícil desarrollo (fastidiosos).

\section{¿Cómo ejercen su acción antibacteriana estas nuevas quinolonas?}

Así como sus antecesoras, mediante la inhibición de la síntesis del ADN bacteriano. El cromosoma bacteriano, anillo único de doble hebra (ADN), en la mayoría de las bacterias se mantiene en un estado energéticamente sobreenrollado, forma requerida para procesos celulares críticos como son la replicación y transcripción.

Dos enzimas estructuralmente homólogas participan en este estado de sobreenrollamiento, la ADN girasa o topoisomerasa II responsable de la mantención del sobreenrollamiento y la topoisomerasa IV responsable de la separación de las hebras hijas durante la división celular; ambas son blanco de acción de las quinolonas. La inhibición enzimática resulta en una rápida muerte de la célula bacteriana. Su acción sobre microorganismos Gram negativos tiene por blanco primario de acción, la topoisomerasa II. En su acción sobre las cocáceas Gram positivas en cambio, resulta primordial la inhibición de la topoisomerasa IV. La especificidad del blanco da las variaciones de acción antimicrobiana de este grupo. , $^{2,3}$

\section{Actividad antibacteriana en el grupo de nuevas quinolonas}

Las quinolonas son una clase de antimicrobianos con actividad típicamente bactericida frente a un gran número de especies bacterianas, su CBM es igual o sólo el doble de su CIM.

Hemos mencionado que una necesidad que debían cubrir esta nuevas quinolonas es aumentar la actividad frente a bacterias Gram positivas, que en los últimos años se han convertido en un problema para microbiólogos y clínicos: S. pneumoniae, Streptococcus grupo viridans, Enterococcus resistentes y SAMR.

\section{Bacterias Gram positivas}

La actividad sobre $S$. pneumoniae ha sido un gran logro en el grupo de nuevas quinolonas, reemplazando la nula o escasa actividad sobre este patógeno de sus predecesores. 
Aspectos microbiológicos de las nuevas quinolonas - O. Trucco A.

Tabla 1. Actividad de nuevas quinolonas frente a Streptococcus pneumoniae $\left(\mathrm{CIM}_{90} \mu \mathrm{g} / \mathrm{ml}\right)$

\begin{tabular}{lcc}
\hline & Rango CIM $_{\mathbf{9 0}} \boldsymbol{\mu g} / \mathbf{m l}$ & Adaptado de \\
\hline Trovafloxacina & $0,006-0,25$ & Ref 11 \\
Sparfloxacina & $0,25-1$ & \\
Ciprofloxacina & $1-8$ & Ref 4 \\
Grepafloxacina & $0,06-0,5$ & \\
Ciprofloxacina & $0,5-8,0$ & Ref 13 \\
Levofloxacina & $0,25-2$ & \\
Ciprofloxacina & $1-8$ &
\end{tabular}

Los estudios de $\mathrm{CIM}_{90}$ que comparan la actividad de ellas frente a ciprofloxacina, demuestran en las primeras, un aumento hasta diez veces en su actividad antineumocóccica, siendo trovafloxacina la de mejor actividad. Estos valores son independientes de la CIM de este patógeno para penicilina, resultando igualmente activas frente a cepas sensibles o resistentes (sea ésta intermedia o elevada resistencia)., ${ }^{4}$ No se han descrito cepas resistentes que sobrepasen el valor de corte de $2 \mu \mathrm{g} / \mathrm{ml}$. (Tabla 1 ).

Los estudios demuestran que la actividad in vitro de levofloxacina y sparfloxacina supera en dos veces la documentada en ciprofloxacina; grepafloxacina y trovafloxacina demuestran aún mejor actividad siendo esta 8 a 10 veces superior.

Frente a otras especies de Streptococcus, las nuevas quinolonas demuestran también mejor actividad que ciprofloxacina, con $\mathrm{CIM}_{90}$ entre 0,06 y $0,5 \mu \mathrm{g} / \mathrm{ml}$ versus 0,5 a $4 \mu \mathrm{g} / \mathrm{ml}$ frente a $S$. pyogenes. Aunque superan también a ciprofloxacina en su actividad frente a Enterococcus, en la mayoría de los estudios in vitro la $\mathrm{CIM}_{90}$ oscila entre 0,25 y $8 \mu \mathrm{g} / \mathrm{ml}$ para cepas de E. faecalis con cifras superiores e igual o mayor a $8 \mu \mathrm{g} / \mathrm{ml}$ frente a E. faecium (todas cepas sensibles a vancomicina). ${ }^{5}$ En la Tabla 2 se observa la actividad comparativa entre trovafloxacina, sparfloxacina y ciprofloxacina.

Frente a $S$. aureus las nuevas quinolonas han demostrado mejor actividad antibacteriana que sus predecesoras (ciprofloxacina) cuando se trata de cepas sensibles a oxacilina, actividad superior de dos a ocho veces. Esta actividad es menor en cepas de SAMR, resistentes a ciprofloxacina. (Tabla 3)

Tabla 2. Actividad in vitro de nuevas quinolonas sobre diversas especies de Streptococcus $\left(\mathrm{CIM}_{90} \mu \mathrm{g} / \mathrm{ml}\right)$

\begin{tabular}{lccc}
\hline & $\begin{array}{c}\text { S. pyogenes } \\
(\mathbf{n : ~ 2 4 2 )}\end{array}$ & $\begin{array}{c}\text { E. faecalis } \\
(\mathbf{n : ~ 5 4 9 )}\end{array}$ & $\begin{array}{c}\text { E. faecium } \\
(\mathbf{n : 1 2 0})\end{array}$ \\
\hline Trovafloxacina & $0,06-0,5$ & $0,25-8$ & $1-4$ \\
Ciprofloxacina & $0,5-4$ & $1-32$ & $2-8$ \\
Sparfloxacina & $0,4-2$ & $0,5-1$ & 1 \\
\hline
\end{tabular}

Tabla 3. Actividad in vitro de nuevas quinolonas frente a Staphylococcus aureus $\left(\mathrm{CIM}_{90} \mu \mathrm{g} / \mathrm{ml}\right)$

\begin{tabular}{llrl}
\hline & S. aureus & SAMR & Adaptado de \\
\hline Trovafloxacina & $0,015-0,5$ & $1-8$ & Ref 5 \\
Sparfloxacina & $0,06-0,25$ & $8-16$ & \\
Ciprofloxacina & $0,25-8$ & $16-128$ & \\
Grepafloxacina & 0,12 & 4 & JAC 33: 283-8 \\
Ciprofloxacina & 1 & 8 & \\
Levofloxacina & $0,06-0,5$ & 16 & AAC 1994; 38: 2702-9 \\
Ciprofloxacina & $0,12-2$ & 64 & \\
\hline
\end{tabular}




\section{Bacterias Gram negativas}

En los bacilos Gram negativos del grupo de enterobacterias, los estudios comparativos de la actividad in vitro de las nuevas moléculas versus sus antecesoras, demuestran que todas las quinolonas ensayadas poseen buena actividad frente a estos microorganismos, con excepción de cepas de Serratia sp y Providencia $s p$ (donde demuestran escasa actividad).

Las diferencias en CIM entre las quinolonas no superó las dos diluciones. Frente a $P$. aeruginosa tampoco es importante el aporte de las nuevas quinolonas, donde demuestran variada pero similar o inferior actividad a ciprofloxacina. Los restantes bacilos Gram negativos no fermentadores son inhibidos por concentraciones menores de los nuevos compuestos de este grupo de antimicrobianos. ${ }^{6,7}$

En patógenos respiratorios fastidiosos como H. influenzae y $M$. catarrhalis, la actividad de las nuevas quinolonas es sólo ligeramente superior a sus predecesoras. Resultan igualmente activas en cepas productoras o no de $\beta$ lactamasas. ${ }^{8,9}$ (Tabla 4).

Frente a $N$. gonorrhoeae las fluoroquinolonas demuestran excelente actividad in vitro con CIMs muy pequeñas, del orden de $0,004 \mu \mathrm{g} / \mathrm{ml}$; este grupo de nuevas moléculas permite cubrir cepas resistentes a ciprofloxacina, sean estas resistentes o no a penicilina.

$M$. pneumoniae, $C$. pneumoniae y $L$. pneumophila son inhibidas por pequeñas concentraciones de las quinolonas de tercera generación, siendo cuatro veces más activas que sus predecesoras; la mayor actividad se demuestra frente a especies de legionelas. ${ }^{10,11}$ (Tabla 5).

\section{Bacterias anaerobias estrictas}

Las quinolonas disponibles hoy son inactivas frente a especies anaerobias estrictas. Levofloxacina y grepafloxacina permanecen inactivas frente a este grupo de microorganismos, trovafloxacina en cambio tiene como su mayor ventaja su acción anti anaerobia. Este antimicrobiano es 32 veces más activo que

Tabla 4. Actividad in vitro de nuevas quinolonas frente a

Haemophilus influenzae y Moraxella catarrhalis $\left(\mathrm{CIM}_{90} \mu \mathrm{g} / \mathrm{ml}\right)$

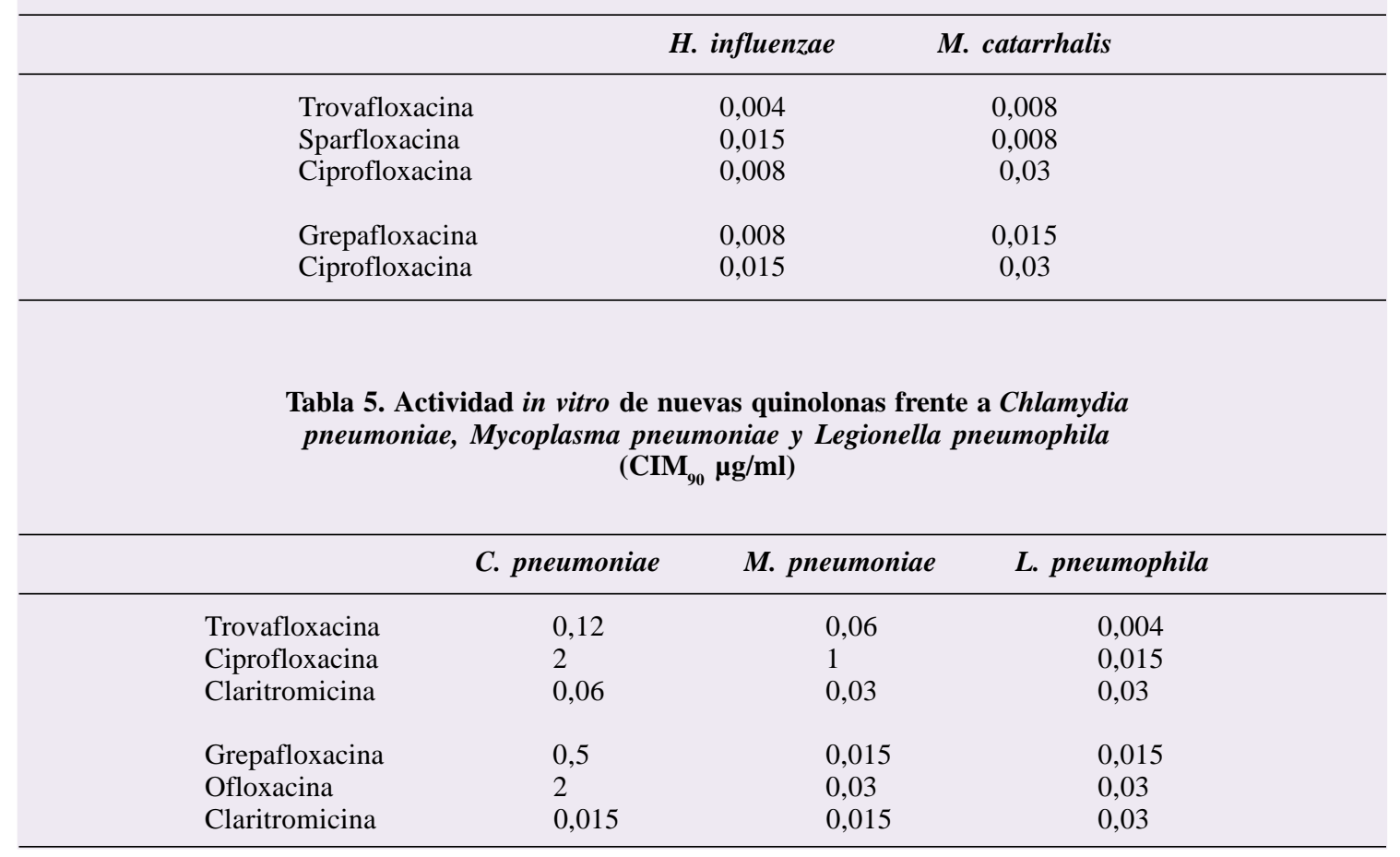


Aspectos microbiológicos de las nuevas quinolonas - O. Trucco A.

Tabla 6. Actividad in vitro de nuevas quinolonas frente a especies anaerobias estrictas $\left(\mathrm{CIM}_{90} \mu \mathrm{g} / \mathrm{ml}\right)$

\begin{tabular}{lcccc}
\hline & Bacteroides sp & Prevotella $\boldsymbol{s p}$ & Fusobacterium $\boldsymbol{s p}$ & Clostridium $\boldsymbol{s p}$ \\
\hline Trovafloxacina & $0,25-0,5$ & $1-2$ & $0,5-1,0$ & $0,03-0,25$ \\
Sparfloxacina & $2-8$ & $2-4$ & $0,5-1,0$ & $0,06-0,32$ \\
Ciprofloxacina & $16-32$ & $4-32$ & $2-8$ & $0,32-0,5$ \\
Metronidazol & $1-4$ & $4-8$ & $2-4$ & $2-4$ \\
\hline
\end{tabular}

ciprofloxacina frente a Bacteroides $s p$, siendo bactericida a menos de $1 \mu \mathrm{g} / \mathrm{ml}$ en casi todas las especies anaerobias. Sparfloxacina demuestra también actividad anti anaerobia, inferior a la demostrada en trovafloxacina. ${ }^{12,13}$ (Tabla 6).

Podemos concluir que las quinolonas de tercera generación cumplieron los desafíos propuestos, mejoraron la actividad frente a bacterias Gram positivas previamente no cubiertas por este grupo de antimicrobianos; S. pneumoniae incluyendo cepas resistentes a penicilina, superaron la actividad frente a SAMR o SAMS, al igual que frente a otras especies de Streptococcus no neumococo y Enterococcus $s p$. Algunas de las nuevas quinolonas demostraron buena actividad frente a microorganismos anaerobios, limitación de las quinolonas disponibles hoy.

En el grupo de bacterias Gram negativas, mantuvieron buena actividad frente a enterobacterias y no fermentadores, superaron a ciprofloxacina frente a algunos microorganismos fastidiosos, como también en su actividad frente a patógenos atípicos.

\section{¿Resistencia bacteriana frente a nuevas quinolonas?}

La resistencia a todas las quinolonas es cromosomal y puede ocurrir por etapas (una o más mutaciones). Microorganismos altamente susceptibles pueden permanecer sensibles con sólo una mutación en su genoma, en otros en cambio, dicha mutación puede determinar su resistencia.

Tres son los mecanismos de resistencia bacteriana descritos en este grupo de antimicrobianos: $:^{14,15}$
- La alteración de las enzimas blanco es el factor de resistencia más importante. Mutaciones en el gen gyrA que codifica la subunidad A de la ADNgirasa: región determinante de la resistencia en quinolonas o quinolone resistance determinant region (QRDR), resulta en un aumento de la CIM de todas las quinolonas. Mutaciones adicionales en el gen parC que codifica la topoisomerasa IV, parecen ser importantes en la resistencia de los microorganismos Gram positivos.

- Mutaciones que llevan a disminución en la expresión de porinas de la membrana externa de bacterias Gram negativas, resultan en disminución de la acumulación de quinolona y así disminución de la susceptibilidad.

- Un mecanismo de eflujo dependiente de energía, por el cual la quinolona es activamente eliminada fuera de la célula bacteriana, mutación del gen norA, también ha sido caracterizado tanto en bacterias Gram negativas como positivas.

Entre cepas de patógenos respiratorios como $S$. pneumoniae ha sido descrita, muy excepcionalmente, resistencia a nuevas fluoroquinolonas, aún en cepas resistentes a penicilina y multiresistentes. Frente a otros microorganismos Gram positivos como Streptococcus $\beta$ hemolíticos, no se han descrito cepas resistentes. Enterococcus sp y $S$. aureus demuestran porcentajes variables de sensibilidad frente a este grupo de antibióticos, 61 a $92 \%$ en el primero y 85 a $100 \%$ en cepas de Staphylococcus oxacilina susceptibles. En cepas resistentes a meticilina, el porcentaje de microorganismos sensibles es menor con cifras entre 17 y 69\%. En el grupo de patógenos Gram positivos, cepas resistentes a ciprofloxa- 
cina pueden ser entonces susceptibles a estas quinolonas ya que se necesita doble mutación en ambas topoisomerasas para dar resistencia y eso ocurre infrecuentemente, en tasa de $10 \% .{ }^{14}$

En el grupo de enterobacterias y bacilos no fermentadores, en que se describe la adquisición de resistencia cruzada a las fluoroquinolonas actualmente disponibles, la actividad de las nuevas quinolonas es excelente con bajos porcentajes de resistencia (1 a 4\%); mayor número de cepas resistentes se describen en $P$. aeruginosa. Completa susceptibilidad ha sido descrita en patógenos respiratorios como $H$. influenzae y $M$. catarrhalis.

Las nuevas fluoroquinolonas serán sin duda un aporte para el manejo de ciertas patologías infecciosas por su espectro de actividad antimicrobiana y propiedades farmacocinéticas; la experiencia clínica permitirá redondear el marco de uso de este nuevo grupo de antimicrobianos.

\section{RESUMEN}

Las nuevas quinolonas "de tercera generación": levofloxacina, sparfloxacina, trovafloxacina y grepafloxacina se caracterizan por mayor actividad in vitro que sus predecesoras sobre Streptococcus pneumoniae, Streptococcus pyogenes y Staphylococcus aureus sensible a meticilina, conservan actividad sobre bacilos Gram negativos entéricos (pero menos que ciprofloxacina), también son activas sobre agentes causales de neumonías atípicas (Mycoplasma pneumoniae, Chlamydia pneumoniae, Legionella pneumoniae) y en algunos casos, sobre especies anaerobias estrictas (trovafloxacina, sparfloxacina).

Considerando los avances en espectro antibacteriano, constituirán en los próximos años un aporte significativo en el manejo de algunas patologías infecciosas ambulatorias y de manejo intrahospitalario.

\section{BIBLIOGRAFIA}

1.- NEU H. The quinolones. Infect Dis Clin North Am 1989; 3: 625-39.

2.- WIEDEMAN B, HEISING P. Antibacterial activity of grepafloxacin. J Antimicrob Chemother 1997; 40: $19-25$.

3.- PAN X, FISHER L. Targeting of DNA gyrase in Streptococcus pneumoniae by sparfloxacin selective targeting of gyrase or topoisomerase IV by quinolones. Antimicrob Agents Chemother 1997; 41: 471-4.

4.- GOLDSMITH C, MOORE J, MURPHY P. Pneumococcal resistance in the UK. J Antimicrob Chemother 1997; 40: 11-8.

5.- BRIGHTY K, GOOTZ T. The chemistry and biological profile of trovafloxacin. $\mathrm{J}$ Antimicrob Chemother 1997; 39: 1-14.

6.- CHILD J, ANDREWS J, BOSWELL F, BREENWALD N. The in vitro activity of CP 99219 a new phtyridone antimicrobial agent comparison with fluoquinolones agents. J Antimicrob Chemother 1995; 35: 869-76.

7.- NEU H, CHIN C. In vitro activity of the newer fluoroquinolones CP 99219. Antimicrob Agents Chemother 1994; 38: 15-22.

8.- WISW R, ANDREUS J. The ability of grepafloxacin against respiratory pathogens in the UK. J Antimicrob Chemother 1997; 40: 27-30.

9.- FINCH R. The role of new quinolones in the treatment of respiratory tract infections. Drugs 1995; 49: 1-3.

10.- MIDGWAY G, SALMAN H, ROBBINS M, DENCER C. The in vitro activity of grepafloxacin against Chlamydia spp, Mycoplasma spp, Ureaplasma spp and Legionella spp. J Antimicrob Chemother 1997; 40: 31-4.

11.- FELMINGHAM D, ROBBINS M, DUGLEY K, MATHIAS L. In vitro activity of trovafloxacin, a new fluoroquinolone against recent clinical isolates. J Antimicrob Chemother 1997; 39: 43-9.

12.- SPANGLER S, JACOBS M, APPELBAUM P. Time killing study of the activity of trovafloxacin compared with ciprofloxacin, metronidazol, cefoxitin, piperacillin, piperacillin-tazobactam against six anaerobes. J Antimicrob Chemother 1997; 39: 23-7.

13.- WEXLER H, MOLITORIS G, MOLITORIS D, FINEGOLD S. In vitro activity of levofloxacin against a selected group of anaerobic bacteria isolated from skin and soft tissue infections. Antimicrob Agent Chemother 1998; 42: 984-6.

14.- WIEDERMAN B, HEISING P. Mechanism of quinolone resistance. Infection 1994; 22: 73-9.

15.- HEISING P, SCHEDIETZKY H, FALKENSTEIN P. Mutations in the gyr $A$ gene of a high level fluoroquinolone resistant clinical isolates of $E$. coli. Antimicrob Agents Chemother 1993; 37: 696-701.

Correspondencia a:

Olivia Trucco Aray

Condell 303, Santiago 9, Chile

Fono/Fax: 56 (2) 2045460.

Email: otrucco@machi.med.uchile.cl 